\title{
EIGENVALUE PROBLEM OF BIHARMONIC EQUATION WITH HARDY POTENTIAL
}

\author{
Yangxin Yao, Shaotong He, and Qingtang Su
}

\begin{abstract}
In this paper, we consider the eigenvalue problem of biharmonic equation with Hardy potential. We improve the results of references by introducing a new Hilbert space.
\end{abstract}

\section{Introduction}

In 2006, Adimurthi, M. Grossi, and S. Santra [2] proved that, if $0 \in \Omega \subset$ $B_{R}(0)$ is a bounded domain in $\mathbb{R}^{4}$, and $R>0, R_{1}>e R$, then $\forall u \in H_{0}^{2}(\Omega)$ or $\forall u \in H^{2}(\Omega) \cap H_{0}^{1}(\Omega)$, we have

(1)

$$
\int_{\Omega}|\triangle u|^{2} \mathrm{~d} x-\int_{\Omega} \frac{u^{2}}{|x|^{4}\left(\ln R_{1} /|x|\right)^{2}} \mathrm{~d} x \geq \sum_{i=2}^{\infty} \int_{\Omega} \frac{u^{2}}{|x|^{4}\left(\ln R_{1} /|x|\right)^{2}} X_{2}^{2} \cdots X_{i}^{2} \mathrm{~d} x,
$$

where -1 is the best constant and can't be achieved by any nontrival function $u \in H_{0}^{2}(\Omega)$ or $\forall u \in H^{2}(\Omega) \cap H_{0}^{1}(\Omega)$, where

$$
X_{i}(x):=Y_{i}\left(\frac{|x|}{R_{1}}\right), i=1,2,3, \ldots
$$

and

$$
\begin{gathered}
Y_{1}(t):=(1-\ln t)^{-1}, t \in(0,1], \\
Y_{i}(t):=Y_{i-1}\left(Y_{1}(t)\right), t \in(0,1], i=2,3,4 \ldots, \\
Y_{i}(0)=0, Y_{i}(1)=1,0 \leq Y_{i}(t) \leq 1 .
\end{gathered}
$$

Furthermore, if we define

$$
\lambda(\Omega)=\inf _{u \in H_{0}^{2}(\Omega)}\left\{\int_{\Omega}|\Delta u|^{2} \mathrm{~d} x-\int_{\Omega} \frac{u^{2}}{|x|^{4}(\ln R /|x|)^{2}} \mathrm{~d} x \mid \int_{\Omega} u^{2} \mathrm{~d} x=1\right\},
$$

Received December 25, 2008.

2000 Mathematics Subject Classification. 35J40, 35J60, 35J85.

Key words and phrases. biharmonic equations, Hardy type inequality, maximum principle.

Supported by NSFC(10771074). 
then $\lambda(\Omega)$ can't be achieved by any domain $\Omega$. This means that the following eigenvalue problem

$$
\begin{cases}\triangle^{2} u-\frac{u}{|x|^{4}(\ln R /|x|)^{2}}=\lambda u & x \in \Omega \\ u \neq 0 & x \in \Omega \\ u \in H_{0}^{2}(\Omega) & \end{cases}
$$

has no solution for $\lambda=\lambda(\Omega)$. Adimurthi, M. Grossi, and S. Santra [2] have considered the following eigenvalue problem

$$
\begin{cases}\triangle^{2} u-\frac{q(x) u}{|x|^{4}(\ln R /|x|)^{2}}=\lambda u & x \in \Omega \\ u \neq 0 & x \in \Omega \\ u \in H_{0}^{2}(\Omega), & \end{cases}
$$

where $0 \leq q(x) \leq 1$. Define

$$
\lambda(q)=\inf _{u \in H_{0}^{2}(\Omega)}\left\{\int_{\Omega}|\triangle u|^{2} \mathrm{~d} x-\int_{\Omega} \frac{q(x) u^{2}}{|x|^{4}(\ln R /|x|)^{2}} \mathrm{~d} x \mid \int_{\Omega} u^{2} \mathrm{~d} x=1\right\}
$$

if $N=4$, and $q(x)$ satisfies the following assumptions, they have the following interesting results:

(i) If $q(x)$ satisfies

$$
\liminf _{x \rightarrow 0}(\ln \ln R /|x|)^{2}(1-q(x))>3,
$$

then $\lambda(q)$ is achieved by $u$, and (3) has solutions for $\lambda=\lambda(q)$. Furthermore, if $\Omega$ is a unit ball centered with the origin, we can choose $u>0$.

(ii) If $\Omega$ is a unit ball centered with the origin, then $\lambda(q)$ is not achieved by any non-negative function, provided $q(x)$ satisfies

$$
\sup _{0<|x| \leq R_{1}}(\ln \ln R /|x|)^{2}(1-q(x)) \leq 3
$$

for some $0<R_{1}<1$.

For the case $N \geq 5$, A. Tertikas and N. Zographopolous [6] have proved the following inequality

(4)

$$
\int_{\Omega}\left(|\triangle u|^{2}-\frac{N^{2}(N-4)^{2}}{16} \frac{|u|^{2}}{|x|^{4}}\right) \mathrm{d} x \geq\left(1+\frac{N(N-4)}{8}\right) \int_{\Omega} \frac{u^{2}}{|x|^{4}(\ln R /|x|)^{2}} \mathrm{~d} x
$$

which holds for any $u \in H_{0}^{2}(\Omega)$, where $R>\sup _{x \in \Omega}|x|$. If we define $\lambda_{N}(\Omega)$ as

$$
\lambda_{N}(\Omega)=\inf _{u \in H_{0}^{2}(\Omega)}\left\{\int_{\Omega}\left(|\triangle u|^{2}-\frac{N^{2}(N-4)^{2}}{16} \frac{|u|^{2}}{|x|^{4}}\right) \mathrm{d} x \mid \int_{\Omega} u^{2} \mathrm{~d} x=1\right\},
$$


then $\lambda_{N}(\Omega)$ is not achieved by any domain $\Omega[6]$. This means that the following eigenvalue problem

$$
\begin{cases}\triangle^{2} u-\frac{N^{2}(N-4)^{2}}{16} \frac{u}{|x|^{4}}=\lambda u & x \in \Omega \\ u \neq 0 & x \in \Omega \\ u \in H_{0}^{2}(\Omega) & \end{cases}
$$

has no solution for $\lambda=\lambda_{N}(\Omega)$. Adimurthi, M. Grossi, and S. Santra [2] considered the following problem

$$
\begin{cases}\triangle^{2} u-\frac{N^{2}(N-4)^{2}}{16} \frac{q(x) u}{|x|^{4}}=\lambda u & x \in \Omega \\ u \neq 0 & x \in \Omega \\ u \in H_{0}^{2}(\Omega), & \end{cases}
$$

where $q \in C^{0}(\bar{\Omega}), 0 \leq q(x) \leq 1$. Let

$$
\lambda_{N}(q)=\inf _{u \in H_{0}^{2}(\Omega)}\left\{\int_{\Omega}|\Delta u|^{2} \mathrm{~d} x-\frac{N^{2}(N-4)^{2}}{16} \int_{\Omega} \frac{q(x) u^{2}}{|x|^{4}} \mathrm{~d} x \mid \int_{\Omega} u^{2} \mathrm{~d} x=1\right\} .
$$

They get the following interesting results:

(i) $\lambda_{N}(q)$ is achieved for some function $u$ in $H_{0}^{2}(\Omega)$, and (6) has solutions for $\lambda=\lambda_{N}(q)$ if $q(x)$ satisfies

$$
\liminf _{x \rightarrow 0}(\ln 1 /|x|)^{2}(1-q(x))>\frac{6\left(N^{2}-4 N+8\right)}{N^{2}(N-4)^{2}} .
$$

Furthermore, if $\Omega$ is a unit ball centered with the origin, then we can choose $u>0$.

(ii) If $\Omega$ is a unit ball centered with the origin, then $\lambda_{N}(q)$ can't be achieved if $q(x)$ satisfies

$$
\sup _{0<|x| \leq R_{2}}(\ln 1 /|x|)^{2}(1-q(x)) \leq \frac{6\left(N^{2}-4 N+8\right)}{N^{2}(N-4)^{2}}
$$

for some $0<R_{2}<1$.

It seems that (7) and (8) can not be improved since they have given an almost sufficient and necessary condition. Observe that if $q(x) \equiv 1$, the eigenvalue problems (3) and (6) have no non-trivial solution in $H_{0}^{2}(\Omega)$. So our first consideration is to weaken the assumption of $q(x)$ so that the result of Adimurthi in [2] can be improved.

Actually, we can achieve this. We find that, if we consider the above problems in a new Hilbert space, whose norm is not equivalent to that of $H_{0}^{2}(\Omega)$, the assumption of $q(x)$ can be weaken.

Furthermore, we pay more attention to the eigenvalue problems with two Hardy potential. 
(1) Let $N \geq 5$. We consider the following problem:

$$
\begin{cases}\triangle^{2} u-\frac{N^{2}(N-4)^{2}}{16} \frac{u}{|x|^{4}}-\mu_{1} \frac{q(x) u}{|x|^{4}(\ln R /|x|)^{2}}=\lambda \eta(x) u & x \in \Omega \\ u \neq 0 & x \in \Omega \\ u=\frac{\partial u}{\partial \gamma}=0 & x \in \partial \Omega\end{cases}
$$

where $0 \leq \mu_{1} \leq 1+N(N-4) / 8$.

(2) Let $N=4$. We consider the weighted eigenvalue problem with two Hardy potential as follow:

(10)

$$
\begin{cases}\triangle^{2} u-\frac{u}{|x|^{4}(\ln R /|x|)^{2}}-\mu_{2} \frac{q(x) u}{|x|^{4}(\ln R /|x|)^{2}(\ln \ln R /|x|)^{2}}=\lambda \eta(x) u & x \in \Omega \\ u \neq 0 & x \in \Omega \\ u=\frac{\partial u}{\partial \gamma}=0 & x \in \partial \Omega,\end{cases}
$$

where $0 \leq \mu_{2} \leq 1$.

For $(9), \mu_{1}=1+N(N-4) / 8$ is the best constant of inequality (4) in the right hand side. In this case, the singular term $1 /\left(|x|^{4}(\ln R /|x|)^{2}\right)$ is called the critical potential.

For the case $N=4$, no paper has proved that $\mu_{2}=1$ is the best constant of inequality (1) in the right hand side. In this paper, we will give a positive answer that 1 is the best constant. As a result, we are able to identify the critical potential case with the non-critical case.

\section{Main results}

In order to state our main results, we construct a new Hilbert space as follows.

We define $H_{0,1}^{2, N}(\Omega)$ as the completion of $H_{0}^{2}(\Omega)$ with respect to the norm $\|\cdot\|_{H_{0,1}^{2, N}(\Omega)}$, where $\Omega \in R^{N}, N \geq 4$. And the norm $\|\cdot\|_{H_{0,1}^{2, N}(\Omega)}$ be defined as

$$
\|u\|_{H_{0,1}^{2, N}(\Omega)}^{2}= \begin{cases}\int_{\Omega}\left(|\triangle u|^{2}-\frac{u^{2}}{|x|^{4}(\ln R /|x|)^{2}}\right) \mathrm{d} x, & N=4 \\ \int_{\Omega}\left(|\triangle u|^{2}-\frac{N^{2}(N-4)^{2}}{16} \frac{u^{2}}{|x|^{4}}\right) \mathrm{d} x, & N \geq 5\end{cases}
$$

associated with the inner product

$$
a(u, v)= \begin{cases}\int_{\Omega}\left(\Delta u \triangle v-\frac{u v}{|x|^{4}(\ln R /|x|)^{2}}\right) \mathrm{d} x, & N=4 \\ \int_{\Omega}\left(\Delta u \triangle v-\frac{N^{2}(N-4)^{2}}{16} \frac{u v}{|x|^{4}}\right) \mathrm{d} x, & N \geq 5 .\end{cases}
$$


Obviously, the norm $\|\cdot\|_{H_{0,1}^{2, N}(\Omega)}$ is not equivalent to the norm $\|\cdot\|_{H_{0}^{2}}=$ $\left(\int_{\Omega}|\triangle u|^{2} \mathrm{~d} x\right)^{\frac{1}{2}}$. If $1 \leq p<2$, by the $W^{1, p}$ estimation in [2], we have

$$
H_{0}^{2}(\Omega) \subset H_{0,1}^{2, N}(\Omega) \subset W_{0}^{1, p}(\Omega) .
$$

In order to see this, when $N=4$, we give some examples to show this. Consider the function $u(x)=u(|x|)$ defined on $B_{1}(0)$, where

$$
u(r)=(\ln 1 / r)^{a}(\ln \ln 1 / r)^{\delta}
$$

in $B_{R_{0}}(0)$ with $0<R_{0}<e^{-1}$, and smooth up to the boundary on $B_{1}(0) \backslash B_{R_{0}}(0)$. It's easy to check that $u \in H_{0}^{2}(\Omega)$ if and only if $a<1 / 2$, or $a=1 / 2$ and $\delta<-1 / 2$, while $u \in H_{0,1}^{2, N}(\Omega)$ if and only if $a<1 / 2$, or $a=1 / 2$ and $\delta<0$.

If $N \geq 5$, we observe the function $u(x)=u(|x|)$ defined on $B_{1}(0)$, where

$$
u(r)=r^{-\frac{N-4}{2}}(\ln 1 / r)^{a}(\ln \ln 1 / r)^{\delta}
$$

in $B_{R_{0}}(0)$ with $0<R_{0}<e^{-1}$ and smooth up to the boundary on $B_{1}(0) \backslash B_{R_{0}}(0)$. It's easy to check that $u \in H_{0}^{2}(\Omega)$ if and only if $a<-1 / 2$, or $a=-1 / 2$ and $\delta<-1 / 2$, while $u \in H_{0,1}^{2, N}(\Omega)$ if and only if $a<0$, or $a=0$ and $\delta<0$.

Define $L_{\eta}^{2}(\Omega)=\left\{u \mid \int_{\Omega} \eta u^{2} \mathrm{~d} x<\infty\right\}$ with the norm $\|u\|_{L_{\eta}^{2}}=\left(\int_{\Omega} \eta u^{2} \mathrm{~d} x\right)^{1 / 2}$, where $\eta \geq 0$, and for $N \geq 5$,

$$
\limsup _{|x| \rightarrow 0}|x|^{4}(\ln R /|x|)^{2} \eta(x)=0
$$

for $N=4$,

$$
\limsup _{|x| \rightarrow 0}|x|^{4}(\ln R /|x|)^{2}(\ln \ln R /|x|)^{2} \eta(x)=0 .
$$

Obviously, $\eta \equiv 1$ satisfies the above conditions of $\eta$, and $L_{1}^{2}(\Omega)=L^{2}(\Omega)$.

we mainly deal with the following problems:

- Some related theorems about the new Hilbert space $H_{0,1}^{2, N}(\Omega)$, including the embedding theorem, maximum principle, etc.

- As an application of $H_{0,1}^{2, N}(\Omega)$, we consider the eigenvalue problem (9) as well as (10), and find the existence of solutions and positive solutions.

(1) For $N \geq 5$, we consider the eigenvalue problem with two singular terms as problem (9), where $\eta \geq 0, \eta \in L^{\infty}\left(\Omega \backslash B_{r}(0)\right), \forall r>0$, and $\eta$ satisfies (11). Define

$$
\lambda_{\mu_{1}}(q)=\inf _{u \in H_{0,1}^{2, N}(\Omega)}\left\{I_{\mu_{1}}(u) \mid \int_{\Omega} \eta(x) u^{2} \mathrm{~d} x=1\right\},
$$

where

$$
I_{\mu_{1}}(u)=\int_{\Omega}\left(|\triangle u|^{2}-\frac{N^{2}(N-4)^{2}}{16} \frac{u^{2}}{|x|^{4}}-\mu_{1} \frac{q(x) u^{2}}{|x|^{4}(\ln R /|x|)^{2}}\right) \mathrm{d} x .
$$


(2) Similarly, for the case of $N=4$, we discuss the eigenvalue problem (10), where $\eta \geq 0, \eta \in L^{\infty}\left(\Omega \backslash B_{r}(0)\right), \forall r \geq 0$, and $\eta$ satisfies (12). We define

$$
\tau_{\mu_{2}}(q)=\inf _{u \in H_{0,1}^{2, N}(\Omega)}\left\{J_{\mu_{2}}(u) \mid \int_{\Omega} \eta(x) u^{2} \mathrm{~d} x=1\right\},
$$

where

$$
J_{\mu_{2}}(u)=\int_{\Omega}\left(|\triangle u|^{2}-\frac{u^{2}}{|x|^{4}(\ln R /|x|)^{2}}-\mu_{2} \frac{q(x) u^{2}}{|x|^{4}(\ln R /|x|)^{2}(\ln \ln R /|x|)^{2}}\right) \mathrm{d} x .
$$

Remark 2.1. It's easy to check that the functionals $I_{\mu_{1}}, J_{\mu_{2}}\left(\mu_{1}<1+N(N-\right.$ $\left.4) / 8, \mu_{2}<1\right)$ are coercive on $H_{0,1}^{2, N}(\Omega)$. It's also easy to find that $J_{\mu_{1}}, I_{\mu_{2}}$ are weak lower semicontinuous and lower bounded. However, we should be aware that when $\mu_{1}=1+N(N-4) / 8, \mu_{2}=1$, the functionals $I_{\mu_{1}}, J_{\mu_{2}}$ are not coercive on $H_{0,1}^{2, N}(\Omega)$.

The main result of this paper is as follows:

Theorem 2.1. Let $N \geq 5,0 \leq \mu_{1} \leq 1+N(N-4) / 8, q \in C^{0}(\bar{\Omega}), 0 \leq q(x) \leq$ $1, \eta(x) \geq 0, \eta(x) \in L^{\infty}\left(\Omega \backslash B_{r}(0)\right), \forall r>0$, and $\eta$ satisfies (11). Then

(1) If $0 \leq \mu_{1}<1+N(N-4) / 8, \lambda_{\mu_{1}}(q)$ can be achieved and problem (9) has a nontrivial solution $u \in H_{0,1}^{2, N}(\Omega)$. Furthermore, if $\Omega$ is a unit ball centered with the origin, then we can choose $u>0$ on $\Omega$.

(2) If $\mu_{1}=1+N(N-4) / 8$, and $q(x)$ satisfies the extra condition

$$
\limsup _{|x| \rightarrow 0} q(x)=0
$$

then $\lambda_{\mu_{1}}(q)$ can be achieved and problem (9) has a nontrivial solution $u \in$ $H_{0,1}^{2, N}(\Omega)$. Furthermore, if $\Omega$ is a unit ball centered with the origin, then we can choose $u>0$ on $\Omega$.

Similar to Theorem 2.1, for the case of $N=4$, we have the following theorem:

Theorem 2.2. Suppose that $N=4,0 \leq \mu_{2} \leq 1, q \in C^{0}(\bar{\Omega}), 0 \leq q(x) \leq$ $1, \eta(x) \geq 0, \eta(x) \in L^{\infty}\left(\Omega \backslash B_{r}(0)\right)$ for any $r>0$, and $\eta$ satisfies (12). Then

(1) If $0 \leq \mu_{2}<1, \tau_{\mu_{2}}(q)$ can be achieved and problem (10) has nontrivial solutions $u \in H_{0,1}^{2, N}(\Omega)$.

(2) If $\mu_{2}=1$, and $q(x)$ satisfies the extra condition

$$
\limsup _{|x| \rightarrow 0} q(x)=0
$$

then $\tau_{\mu_{2}}(q)$ is achieved and problem (10) has nontrivial solutions $u \in H_{0,1}^{2, N}(\Omega)$. Furthermore, if $\Omega$ is a unit ball centered with the origin, we can choose $u>0$ on $\Omega$. 


\section{Preliminary lemmas}

Lemma 3.1. The Hilbert space $H_{0,1}^{2, N}(\Omega)$ is embedded into $L_{\eta}^{2}(\Omega)$ and the embedding is compact, where $\eta \geq 0$, if $N \geq 5$, then $\eta$ satisfies (11), while $N=4$ $\eta$ satisfies (12).

Proof. We'll divided the proof into two steps. The first step is to prove that $H_{0,1}^{2, N}(\Omega) \hookrightarrow \hookrightarrow L^{2}(\Omega)$, while the second step is to prove $H_{0,1}^{2, N}(\Omega) \hookrightarrow \hookrightarrow L_{\eta}^{2}(\Omega)$.

Step one: Prove $H_{0,1}^{2, N}(\Omega) \hookrightarrow \hookrightarrow L^{2}(\Omega)$.

From Theorem A.2 of [2], there exist $R_{0}>0, C_{1}>0, C_{2}>0$ such that $\forall R \geq$ $R_{0}, \forall u \in H_{0}^{2}(\Omega)$

$$
\begin{cases}\int_{\Omega}\left(|\Delta u|^{2}-\frac{u^{2}}{|x|^{4}(\ln R /|x|)^{2}}\right) \mathrm{d} x \geq C_{1}\|u\|_{W_{0}^{1, p}(\Omega)}^{2}, & N=4 \\ \int_{\Omega}\left(|\Delta u|^{2}-\frac{N^{2}(N-4)^{2}}{16} \frac{u^{2}}{|x|^{4}}\right) \mathrm{d} x \geq C_{2}\|u\|_{W_{0}^{1, p}(\Omega)}^{2}, \quad N \geq 5\end{cases}
$$

where $1 \leq p<2$. Since $H_{0}^{2}(\Omega)$ is dense in $H_{0,1}^{2, N}(\Omega)$, then the above inequalities are hold for any $u \in H_{0,1}^{2, N}(\Omega)$. It's easy to check that $H_{0,1}^{2, N}(\Omega) \subset W_{0}^{1, p}(\Omega)$, so $H_{0,1}^{2, N}(\Omega) \hookrightarrow W_{0}^{1, p}(\Omega)$. Furthermore, if $p>\frac{2 N}{N+2}$, by Sobolev embedding theorem, the embedding $W_{0}^{1, p}(\Omega) \hookrightarrow L^{2}(\Omega)$ is compact. By [1], $H_{0,1}^{2, N}(\Omega) \hookrightarrow$ $L^{2}(\Omega)$ and the embedding is compact, i.e., $H_{0,1}^{2, N}(\Omega) \hookrightarrow \hookrightarrow L^{2}(\Omega)$.

Step two: Prove $H_{0,1}^{2, N}(\Omega) \hookrightarrow \hookrightarrow L_{\eta}^{2}(\Omega)$.

Since $H_{0,1}^{2, N}(\Omega)$ is a Hilbert space, it's reflexive, and it's separable since $H_{0}^{2}(\Omega)$ is separable and $H_{0,1}^{2, N}(\Omega)$ is dense in $H_{0}^{2}(\Omega)$. By [3], the bounded set of $H_{0,1}^{2, N}(\Omega)$ is weakly compact. Therefore, for any bounded sequence $\left\{u_{n}\right\} \in H_{0,1}^{2, N}(\Omega)$, up to a subsequence, we can assume that

$$
\begin{cases}u_{n} \rightarrow u, & \text { in } H_{0,1}^{2, N}(\Omega) \\ u_{n} \rightarrow u, & \text { in } L^{2}(\Omega)\end{cases}
$$

Since for $N \geq 5, \eta$ satisfies (11), so $\forall \epsilon>0$ small enough, there exists $r>0$, such that $\forall|x|<r,|x|^{4}(\ln R /|x|)^{2} \eta(x)<\epsilon$. Observe that

$$
\begin{aligned}
\int_{\Omega} \eta\left|u_{n}-u\right|^{2} \mathrm{~d} x= & \int_{B_{r}(0)}|x|^{4}(\ln R /|x|)^{2} \eta \frac{\left|u_{n}-u\right|^{2}}{|x|^{4}(\ln R /|x|)^{2}} \mathrm{~d} x \\
& +\int_{\Omega \backslash B_{r}(0)} \eta\left|u_{n}-u\right|^{2} \mathrm{~d} x .
\end{aligned}
$$

Applying (4), $\forall \epsilon>0$, by the above discussion, there exists $r=r(\epsilon)>0$, such that

$$
\int_{B_{r}(0)}|x|^{4}(\ln R /|x|)^{2} \eta \frac{\left|u_{n}-u\right|^{2}}{|x|^{4}(\ln R /|x|)^{2}} \mathrm{~d} x
$$




$$
<\epsilon \int_{B_{r}(0)} \frac{\left|u_{n}-u\right|^{2}}{|x|^{4}(\ln R /|x|)^{2}} \mathrm{~d} x<C \epsilon\left\|u_{n}-u\right\|_{H_{0,1}^{2, N}(\Omega)}^{2} .
$$

Since $\left\{u_{n}\right\}$ is bounded in $H_{0,1}^{2, N}(\Omega)$, letting $\epsilon \rightarrow 0$, we have $\int_{B_{r}(0)} \eta\left|u_{n}-u\right|^{2} \mathrm{~d} x$ $\rightarrow 0$. Moreover,

$$
\int_{\Omega \backslash B_{r}(0)} \eta\left|u_{n}-u\right|^{2} \mathrm{~d} x \leq\|\eta\|_{L^{\infty}\left(\Omega \backslash B_{r}(0)\right)}|| u_{n}-u \|_{L^{2}(\Omega)}^{2} \rightarrow 0, n \rightarrow \infty
$$

therefore $\int_{\Omega} \eta\left|u_{n}-u\right|^{2} \mathrm{~d} x \rightarrow 0$, i.e., $u_{n} \rightarrow u$ in $L_{\eta}^{2}(\Omega)$. If $N=4$, the proof is similar to that of $N \geq 5$. This completes the proof.

Lemma 3.2. Let $N=4$. Then we have

$$
\inf _{u \in H_{0}^{2}(\Omega)} \frac{\int_{\Omega}\left(|\Delta u|^{2}-\frac{u^{2}}{|x|^{4}(\ln R /|x|)^{2}}\right) \mathrm{d} x}{\int_{\Omega} \frac{u^{2}}{|x|^{4}(\ln R /|x|)^{2}(\ln \ln R /|x|)^{2}} \mathrm{~d} x}=1 .
$$

Proof. For any $\epsilon>0$, fix $\delta>0$ and let

$$
u_{\epsilon}(x)= \begin{cases}(\ln R /|x|)^{1 / 2}(\ln \ln R /|x|)^{1 / 2+\epsilon}, & \delta \leq|x| \leq R_{1}<1 \\ a|x|+b, & |x| \leq \delta\end{cases}
$$

and $u_{\epsilon}$ is smooth up to the boundary. To guarantee $u_{\epsilon}$ has a continuous first order derivative on $|x|=\delta$, we require

$$
a=-\frac{1}{2 \delta}(\ln R / \delta)^{-1 / 2}(\ln \ln R / \delta)^{1 / 2+\epsilon}-\frac{1+2 \epsilon}{2 \delta}(\ln R / \delta)^{-1 / 2}(\ln \ln R / \delta)^{-1 / 2+\epsilon}
$$

and

$$
\begin{aligned}
b= & (\ln R / \delta)^{1 / 2}(\ln \ln R / \delta)^{1 / 2+\epsilon}+1 / 2(\ln R / \delta)^{-1 / 2}(\ln \ln R / \delta)^{1 / 2+\epsilon} \\
& +\frac{1+2 \epsilon}{2}(\ln R / \delta)^{-1 / 2}(\ln \ln R / \delta)^{-1 / 2+\epsilon} .
\end{aligned}
$$

Observe that

$$
\begin{aligned}
& \int_{B_{R_{1}}(0)} \frac{u_{\epsilon}^{2}}{|x|^{4}(\ln R /|x|)^{2}(\ln \ln R /|x|)^{2}} \mathrm{~d} x \\
= & 4 \omega_{4} \int_{0}^{R_{1}} \frac{u_{\epsilon}^{2}}{r(\ln R / r)^{2}(\ln \ln R / r)^{2}} \mathrm{~d} r \\
= & 4 \omega_{4} \int_{0}^{\delta} \frac{(a r+b)^{2}}{r(\ln R / r)^{2}(\ln \ln R / r)^{2}} \mathrm{~d} r+4 \omega_{4} \int_{\delta}^{R_{1}} r^{-1}(\ln R / r)^{-1}(\ln \ln R / r)^{-1+2 \epsilon} \mathrm{d} r \\
\triangleq & A+B .
\end{aligned}
$$

For $A$, we have

$$
\begin{aligned}
A & =4 \omega_{4} \int_{0}^{\delta}\left(\frac{a^{2} r}{(\ln R / r)^{2}(\ln \ln R / r)^{2}}+\frac{2 a b}{(\ln R / r)^{2}(\ln \ln R / r)^{2}}+\frac{b^{2}}{r(\ln R / r)^{2}(\ln \ln R / r)^{2}}\right) \mathrm{d} r \\
& \triangleq A_{1}+A_{2}+A_{3} .
\end{aligned}
$$


For any $0 \leq \delta<1$, it's easy to check that $A_{1}, A_{2}, A_{3}$ converge to finite limit as $\epsilon \rightarrow 0$.

For $B$, we have

$$
B=4 \omega_{4} \int_{\delta}^{R_{1}} r^{-1}(\ln R / r)^{-1}(\ln \ln R / r)^{-1+2 \epsilon} \mathrm{d} r \rightarrow \infty(\epsilon \rightarrow 0)
$$

so as $\epsilon \rightarrow 0$, we obtain

$$
\int_{B_{R_{1}(0)}} \frac{u_{\epsilon}^{2}}{|x|^{4}(\ln R /|x|)^{2}(\ln \ln R /|x|)^{2}} \mathrm{~d} x \sim B=4 \omega_{4} \int_{\delta}^{R_{1}} \frac{1}{r \ln R / r(\ln \ln R / r)^{1-2 \epsilon}} \mathrm{d} r .
$$

By direct calculating, if $0 \leq r \leq \delta$, then

$$
\Delta u_{\epsilon}=3 a r^{-1}
$$

while if $\delta<r \leq R_{1}$, then

$$
\begin{aligned}
\Delta u_{\epsilon}= & -r^{-2}(\ln R / r)^{-1 / 2}(\ln \ln R / r)^{1 / 2+\epsilon} \\
& -\frac{1+2 \epsilon}{r^{2}}(\ln R / r)^{-1 / 2}(\ln \ln R / r)^{-1 / 2+\epsilon} \\
& -\frac{1}{4 r^{2}}(\ln R / r)^{-3 / 2}(\ln \ln R / r)^{1 / 2+\epsilon} \\
& +\frac{-1 / 4+\epsilon^{2}}{r^{2}}(\ln R / r)^{-3 / 2}(\ln \ln R / r)^{-3 / 2+\epsilon}
\end{aligned}
$$

and therefore,

$$
\begin{aligned}
& \int_{B_{R_{1}}(0)}\left(\left|\Delta u_{\epsilon}\right|^{2}-\frac{u_{\epsilon}^{2}}{|x|^{4}(\ln R /|x|)^{2}}\right) \mathrm{d} x \\
= & 4 \omega_{4} \int_{0}^{R_{1}}\left(\left|\Delta u_{\epsilon}\right|^{2} r^{3}-\frac{u_{\epsilon}^{2}}{r(\ln R / r)^{2}}\right) \mathrm{d} r \\
= & 36 \omega_{4} \int_{0}^{\delta} a^{2} r \mathrm{~d} r-4 \omega_{4} \int_{0}^{\delta} \frac{(a r+b)^{2}}{r(\ln R / r)^{2}} \mathrm{~d} r \\
& +4 \omega_{4} \int_{\delta}^{R_{1}}\left(\left|\Delta u_{\epsilon}\right|^{2}-r^{-1}(\ln R / r)^{-1}(\ln R / r)^{1+2 \epsilon}\right) \mathrm{d} r \\
\triangleq & D_{1}+D_{2}+D_{3},
\end{aligned}
$$

where

$$
\left\{\begin{array}{l}
D_{1}=36 \omega_{4} \int_{0}^{\delta} a^{2} r \mathrm{~d} r \\
D_{2}=-4 \omega_{4} \int_{0}^{\delta} \frac{(a r+b)^{2}}{r(\ln R / r)^{2}} \mathrm{~d} r \\
D_{3}=4 \omega_{4} \int_{\delta}^{R_{1}}\left(\left|\Delta u_{\epsilon}\right|^{2}-r^{-1}(\ln R / r)^{-1}(\ln R / r)^{1+2 \epsilon}\right) \mathrm{d} r
\end{array}\right.
$$


Obviously, $D_{1}, D_{2}$ converge to finite limit as $\epsilon \rightarrow 0$. For $D_{3}$,

$$
D_{3} \sim 4 \omega_{4} \int_{\delta}^{R_{1}} \frac{1}{r \ln R / r(\ln R / r)^{1-2 \epsilon}} \mathrm{d} r \rightarrow \infty
$$

and therefore

$$
\int_{B_{R_{1}}(0)}\left(\left|\Delta u_{\epsilon}\right|^{2}-\frac{u_{\epsilon}^{2}}{|x|^{4}(\ln R /|x|)^{2}}\right) \mathrm{d} x \backsim 4 \omega_{4} \int_{\delta}^{R_{1}} \frac{1}{r \ln R / r(\ln R / r)^{1-2 \epsilon}} \mathrm{d} r .
$$

Hence, letting $\epsilon \rightarrow 0$, we obtain

$$
\int_{\Omega}\left(\left|\Delta u_{\epsilon}\right|^{2}-\frac{u_{\epsilon}^{2}}{|x|^{4}(\ln R /|x|)^{2}}\right) \mathrm{d} x / \int_{\Omega} \frac{u_{\epsilon}^{2}}{|x|^{4}(\ln R /|x|)^{2}(\ln \ln R /|x|)^{2}} \mathrm{~d} x \rightarrow 1 .
$$

By inequality (1), the proof is completed.

Remark 3.1. By Lemma 3.2, if $\mu_{2}=1$, the singular term

$$
1 /|x|^{4}(\ln R /|x|)^{2}(\ln R /|x|)^{2}
$$

in Theorem 2.2 is called critical potential.

Lemma 3.3. Consider the problem (9), where $0 \leq \mu_{1} \leq 1+N(N-4) / 8$, $\Omega=B$ is a unit ball in $\mathbb{R}^{N}(N \geq 5)$ centered with the origin. If (9) admits a nontrivial solution $u$ for $\lambda=\lambda_{\mu_{1}}(q)$, then $u$ doesn't change sign in $B$.

Proof. The proof is similar to that of Theorem 5.1 of [2]. We will prove it by contradiction. Assume that a solution $u$ of (9) changes sign in $B$, define

$$
K:=\left\{v \in H_{0,1}^{2, N}(B): v \geq 0 \text { a.e., } v=\frac{\partial v}{\partial \gamma}=0 \text { on } \partial B\right\} .
$$

Then $K$ is a close convex cone and $K$ is not empty. So there exists a projection $P: H_{0,1}^{2, N}(B) \rightarrow K$ such that $\forall u \in H_{0,1}^{2, N}(B), \forall v \in K$

$$
a(u-P(u), v-P(u)) \leq 0 .
$$

Since $K$ is a cone, we can replace $v$ with $t v$ in (16), where $t>0$. Letting $t \rightarrow \infty$, we have

$$
a(u-P(u), v) \leq \lim _{t \rightarrow \infty} \frac{1}{t} a(u-P(u), P(u)) .
$$

Hence we have $\Delta^{2}(u-P(u)) \leq 0$, by Boggio's principle, $u-P(u) \leq 0$. Meanwhile, if we replace $v$ with $t P(u)$ in (16), where $t>0$, then we have

$$
(t-1) a(u-P(u), P(u)) \leq 0
$$

so we have $a(u-P(u), P(u))=0$. Therefore $u$ can be divided into $u=u_{1}+u_{2}$, where $u_{1}=P(u) \in K, u_{2}=u-P(u)$, with $u_{2} \leq 0$. It's not hard to check that

$$
\frac{I_{\mu_{1}}\left(u_{1}-u_{2}\right)}{\int_{B} \eta\left|u_{1}-u_{2}\right|^{2} \mathrm{~d} x}<\frac{I_{\mu_{1}}\left(u_{1}+u_{2}\right)}{\int_{B} \eta\left|u_{1}+u_{2}\right|^{2} \mathrm{~d} x}
$$


it contradict with the definition of $\lambda_{\mu_{1}}(q)$. Hence $u$ doesn't change sign in $B$. Since the Green function is strictly positive, so $u$ is strictly positive or negative in $B$.

Similarly we can prove the following theorem.

Lemma 3.4. Consider the problem (10), with $0 \leq \mu_{2} \leq 1$ and $\Omega=B$ is a unit ball in $\mathbb{R}^{4}$ centered with the origin. If (10) admits a nontrivial solution $u$ for $\tau=\tau_{\mu_{2}}(q)$, then $u$ doesn't change sign in $B$.

\section{The proofs of Theorems 2.1, 2.2}

Proof of Theorem 2.1. (1) If $0 \leq \mu_{1}<1+N(N-4) / 8$, then it's easy to check that $I_{\mu_{1}}(u)$ is coercive and weak lower semicontinuous in $H_{0,1}^{2, N}(\Omega)$. Define the manifold

$$
M:=\left\{u \in H_{0,1}^{2, N}(\Omega) \mid \int_{\Omega} \eta u^{2} \mathrm{~d} x=1\right\} .
$$

Then $M$ is a weakly closed subset of $H_{0,1}^{2, N}(\Omega)$. Obviously $M$ is not empty. By [8], $I_{\mu_{1}}(u)$ admits its minimum by a minimizer $u \in M$. So $\lambda_{\mu_{1}}(q)$ is achieved and also the problem (9) has a nontrivial solution. By Lemma 3.3, we can choose their solution $u>0$.

(2) If $\mu_{1}=1+N(N-4) / 8$, the functional $I_{\mu_{1}}(u)$ is not coercive in $H_{0,1}^{2, N}(\Omega)$, so we can not follow the steps of (1). To conquer the difficulty, we consider the following problem:

$$
\begin{cases}\triangle^{2} u-\frac{N^{2}(N-4)^{2}}{16} \frac{u}{|x|^{4}}-\left(1+\frac{N(N-4)}{8}\right) \frac{s q(x) u}{|x|^{4}(\ln R /|x|)^{2}}=\lambda \eta(x) u & x \in \Omega \\ u \neq 0 & x \in \Omega \\ u=\frac{\partial u}{\partial \gamma}=0 & x \in \partial \Omega,\end{cases}
$$

where $0 \leq s<1, q$ and $\eta$ satisfy the assumptions of the theorem. Observe that the operator

$$
\Delta^{2}-\frac{N^{2}(N-4)^{2}}{16} \frac{1}{|x|^{4}}-\left(1+\frac{N(N-4)}{8}\right) \frac{s q(x)}{|x|^{4}(\ln R /|x|)^{2}}
$$

is coercive in $H_{0,1}^{2, N}(\Omega)$. By the first part of the theorem, the above problem admits a nontrivial solution $u_{s}$ for $\lambda_{s}(q)=\lambda_{\mu_{1}}(s q)$. And observe that $\frac{u_{s}}{\left\|u_{s}\right\|_{H_{0,1}^{2, N}}}$ is also a nontrivial solution of (17). Hence $\forall 0 \leq s<1$, we can find $\left\{u_{s}\right\}$ such that $u_{s}$ is a solution of $(17)$ and $\left\|u_{s}\right\|_{H_{0,1}^{2, N}(\Omega)}=1$. Therefore, by Lemma 3.1, up to a subsequence, we have

$$
\begin{cases}u_{s} \rightarrow u_{1}, & \text { in } H_{0,1}^{2, N}(\Omega) \\ u_{s} \rightarrow u_{1}, & \text { in } L_{\eta}^{2}(\Omega)\end{cases}
$$


We will prove that $u_{s} \rightarrow u_{1}$ in $H_{0,1}^{2, N}(\Omega)$ as $s \rightarrow 1$. In the fact, by (17), we have

$$
\begin{aligned}
& \int_{\Omega}\left[\left|\Delta u_{s}\right|^{2}-\frac{N^{2}(N-4)^{2}}{16} \frac{u_{s}^{2}}{|x|^{4}}-\left(1+\frac{N(N-4)}{8}\right) \frac{s q(x) u_{s}^{2}}{|x|^{4}(\ln R /|x|)^{2}}\right] \mathrm{d} x \\
= & \lambda_{s}(q) \int_{\Omega} \eta u_{s}^{2} \mathrm{~d} x .
\end{aligned}
$$

We will verify that, if we take $\omega(x)=\frac{q(x)}{|x|^{4}(\ln R /|x|)^{2}}$, then $\omega$ satisfies the assumption of $\eta$ in the definition of $L_{\eta}^{2}(\Omega)$.

(1) $\forall x \in \Omega, \omega(x) \geq 0$ is obviously;

(2) $\forall x \in \Omega \backslash B_{r}(0)$, we have $\omega(x) \leq r^{-4}$, where $r>0$ and $B_{r}(0) \subset \Omega$. Hence $\omega \in L^{\infty}\left(\Omega \backslash B_{r}(0)\right)$;

(3) Observe that $q(x)$ satisfies (13), we have

$$
\limsup _{|x| \rightarrow 0}|x|^{4}(\ln R /|x|)^{2} \omega(x)=\limsup _{|x| \rightarrow 0} q(x)=0
$$

therefore, $L_{\omega}^{2}(\Omega)$ is well defined. By Lemma $3.1, H_{0,1}^{2, N}(\Omega) \hookrightarrow \hookrightarrow L_{\omega}^{2}(\Omega)$. Hence, we have

$$
\left\{\begin{array}{l}
\int_{\Omega} \frac{q(x) u_{s}^{2}}{|x|^{4}(\ln R /|x|)^{2}} \mathrm{~d} x \rightarrow \int_{\Omega} \frac{q(x) u_{1}^{2}}{|x|^{4}(\ln R /|x|)^{2}} \mathrm{~d} x \\
\int_{\Omega} \eta u_{s}^{2} \mathrm{~d} x \rightarrow \int_{\Omega} \eta u_{1}^{2} \mathrm{~d} x .
\end{array}\right.
$$

Therefore

$$
\begin{aligned}
I_{\mu_{1}}\left(u_{s}\right) & =\int_{\Omega}\left[\left|\Delta u_{s}\right|^{2}-\frac{N^{2}(N-4)^{2}}{16} \frac{u_{s}^{2}}{|x|^{4}}-\left(1+\frac{N(N-4)}{8}\right) \frac{s q(x) u_{s}^{2}}{|x|^{4}(\ln R /|x|)^{2}}\right] \mathrm{d} x \\
& =\lambda_{s}(q) \int_{\Omega} \eta u_{s}^{2} \mathrm{~d} x \rightarrow \lambda_{\mu_{1}}(q) \int_{\Omega} \eta u_{1}^{2} \mathrm{~d} x .
\end{aligned}
$$

By the weak lower semicontinuous of $I_{\mu_{1}}$ and the fact that $\lambda_{s}(q) \rightarrow \lambda_{\mu_{1}}(q)$ as $s \rightarrow 1$, we have

$$
I_{\mu_{1}}\left(u_{1}\right) \leq \liminf _{s \rightarrow 1} I_{\mu_{1}}\left(u_{s}\right)=\lambda_{\mu_{1}}(q) \int_{\Omega} \eta u_{1}^{2} \mathrm{~d} x .
$$

By the definition of $\lambda_{\mu_{1}}(q)$, we have $I_{\mu_{1}}\left(u_{1}\right) \geq \lambda_{\mu_{1}}(q) \int_{\Omega} \eta u_{1}^{2} \mathrm{~d} x$. Therefore, $I_{\mu_{1}}\left(u_{1}\right)=\lambda_{\mu_{1}}(q) \int_{\Omega} \eta u_{1}^{2} \mathrm{~d} x$, and

$$
\begin{aligned}
\left\|u_{s}\right\|_{H_{0,1}^{2, N}(\Omega)}^{2} & =\int_{\Omega}\left(\left|\Delta u_{s}\right|^{2}-\frac{N^{2}(N-4)^{2}}{16} \frac{u_{s}^{2}}{|x|^{4}}\right) \mathrm{d} x \\
& =\left(1+\frac{N(N-4)}{8}\right) \int_{\Omega} \frac{s q(x) u_{s}^{2}}{|x|^{4}(\ln R /|x|)^{2}} \mathrm{~d} x+\lambda_{s}(q) \int_{\Omega} \eta u_{s}^{2} \mathrm{~d} x \\
& \rightarrow\left(1+\frac{N(N-4)}{8}\right) \int_{\Omega} \frac{q u_{1}^{2}}{|x|^{4}(\ln R /|x|)^{2}} \mathrm{~d} x+\lambda_{\mu_{1}}(q) \int_{\Omega} \eta u_{1}^{2} \mathrm{~d} x
\end{aligned}
$$




$$
=\int_{\Omega}\left(\left|\Delta u_{1}\right|^{2}-\frac{N^{2}(N-4)^{2}}{16} \frac{q u_{1}^{2}}{|x|^{4}}\right) \mathrm{d} x=\left\|u_{1}\right\|_{H_{0,1}^{2, N}(\Omega)}^{2} .
$$

Hence $\left\|u_{s}\right\|_{H_{0,1}^{2, N}(\Omega)} \rightarrow\left\|u_{1}\right\|_{H_{0,1}^{2, N}(\Omega)}$, i.e., $u_{s} \rightarrow u_{1}$ in $H_{0,1}^{2, N}(\Omega)$. So $\lambda_{\mu_{1}}(q)$ is achieved by $u_{1}$, and the problem (9) has a nontrivial solution $u_{1}$. By Theorem 3.3 , if $\Omega$ is a unit ball centered with the origin, we can choose $u>0$ or $u<0$. Observe that $-u$ is also a solution of the problem (9), we can choose $u>0$. This completes the proof.

Proof of Theorem 2.2. The proof of this theorem is similar to that of Theorem 2.1 .

\section{References}

[1] R. A. Adams, Sobolev Spaces, Academic Press, New York-London, 1975.

[2] Adimurthi, M. Grossi, and S. Santra, Optimal Hardy-Rellich inequalities, maximum principle and related eigenvalue problem, J. Funct. Anal. 240 (2006), no. 1, 36-83.

[3] I. Ekeland, On the variational principle, J. Math. Anal. Appl. 47 (1974), 324-353.

[4] S. Filippas and A. Tertikas, Optimizing improved Hardy inequalities, J. Funct. Anal. 192 (2002), no. 1, 186-233.

[5] F. Gazzola, H. C. Grunau, and E. Mitidieri, Hardy inequalities with optimal constants and remainder terms, Trans. Amer. Math. Soc. 356 (2004), no. 6, 2149-2168.

[6] A. Tertikas and N. B. Zographopolous, Best constants in the Hardy-Rellich inequalities and related improvements, Adv. Math. 209 (2007), no. 2, 407-459.

[7] M. Willem, Minimax Theorems, Birkhäuser Boston, Inc., Boston, MA, 1996.

[8] Y. X. Yao, Y. T. Shen, and Z. H. Chen, Biharmonic equation and an improved Hardy inequality, Acta Math. Appl. Sin. Engl. Ser. 20 (2004), no. 3, 433-440.

YANGXIN YAO

Department of Mathematics

South China University of Technology

Guangzhou 510640, P. R. China

E-mail address: mayxyao@scut.edu.cn

Shaotong He

Department of Mathematics

South China University of Technology

Guangzhou 510640, P. R. China

E-mail address: 379847218@foxmail.com

Qingtang Su

School of Mathematics and Computational Science

Sun Yat-Sen University

Guangzhou 510275, P. R. China

E-mail address: 656548092@foxmail.com 